\title{
Laser cleaning of oxide iron layer: Efficiency enhancement due to electrochemical induced absorptivity change
}

\author{
P. Pasquet ${ }^{1}$, R. del Coso ${ }^{1}$, J. Boneberg ${ }^{1}$, P. Leiderer ${ }^{1}$, R. Oltra ${ }^{2}$, J.P. Boquillon ${ }^{2}$ \\ ${ }^{1}$ Fakultät für Physik, Universität Konstanz, 78457 Konstanz, Germany \\ (Fax: +49-75/31-88-31-27, E-mail: pasquetp@ hotmail.com) \\ ${ }^{2}$ Laboratoire de Recherches sur la Réactivité des Solides, UMR 5613 CNRS, Laboratoire de Physique UPRES-A 5027 Université, de Bourgogne, \\ BP 47 870, 21078 Dijon Cedex, France
}

Received: 21 July 1999/Accepted: 1 September 1999/Published online: 28 December 1999

\begin{abstract}
Laser cleaning experiments exhibited a higher cleaning efficiency of the laser cleaning upon application of electrochemical potential on an oxidised iron surface. The objective of this study is to clarify the role of such an electrochemical control on cleaning efficiency. Therefore a study by optical spectrometry at normal incidence in the relevant conditions was performed. From these measurements the optical parameters $n$ and $k$ were deduced. While $n$ is mainly not affected, $k$ changes for a factor of 10 upon application of an electrochemical potential. These results explain the differences in laser cleaning efficiency due to the modified absorption length.
\end{abstract}

PACS: 78.20.Ci; 81.65.Cf; 81.65.Mq

Corrosion in industry is a problem for all installations. The classical techniques of surface cleaning are based on mechanical or chemical methods. Thus the substrate may be damaged or polluted. Laser cleaning might be an interesting alternative for this purpose. Two methods were studied up to now in this context, laser ablation of the oxide layer at strong fluence $\left(E \geq 1 \mathrm{~J} / \mathrm{cm}^{2}\right)[1,2]$ and the generation of acoustic waves by laser irradiation at low fluence $\left(E \leq 0.6 \mathrm{~J} / \mathrm{cm}^{2}\right)[3,4]$. The later allows to work below the ablation threshold of the substrate. It was shown that the environment (e.g. gas, liquid, ...) plays an important role on the efficiency of the laser cleaning [5-7]. Furthermore experimental studies [8,9] have shown that laser cleaning on the oxide layer is more efficient when additionally electrochemical potential of suitable sign and size is applied before or during the cleaning process. The aim of this study, is to understand the role of such an electrochemical control on the laser cleaning of an oxidised surface.

\section{Experimental}

The sample preparation in this study started with a pure iron $(99.8 \%)$ substrates. First the surfaces were polished with $1 \mu \mathrm{m}$ diamond powder and rinsed in an ultrasonic bath of

COLA'99 - 5th International Conference on Laser Ablation, July 19-23, 1999 in Göttingen, Germany alcohol and acetone. Then they were placed in an oven at

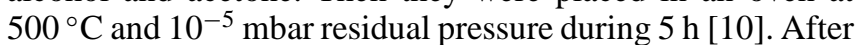
that they were oxidised at $500{ }^{\circ} \mathrm{C}$, for $48 \mathrm{~h}$ with an air residual pressure of 10 mbar [11].

The cleaning has been made in three different conditions, in air for reference, without and with electrochemical control. The irradiation was performed by means of a Nd:YAG laser $(\lambda=532 \mathrm{~nm}, \tau=7 \mathrm{~ns}$, FWHM). Each irradiated area received several laser pulses. Pulse energy densities in the $380-700 \mathrm{~mJ} / \mathrm{cm}^{2}$ ranges were used to irradiate the sample.

For the experiment with electrochemical control, the samples were placed in an electrochemical cell. The electrolyte used, was a solution of $\mathrm{H}_{3} \mathrm{BO}_{3}(0.05 \mathrm{M})$ and $\mathrm{Na}_{2} \mathrm{~B}_{4} \mathrm{O}_{7}$, $10, \mathrm{H}_{2} \mathrm{O}(0.075 \mathrm{M})$ at $\mathrm{pH}=9.2$. A cathodic polarisation $\left(V=-1.55 \mathrm{~V} / \mathrm{Ag} / \mathrm{AgCl} / \mathrm{KCl}_{\text {sat }}\right)$ was applied from 1 to 40 minutes.

The optical study has been made by a spectrometer (Polytec) in combination with a microscope. The wavelengths used were between $450-900 \mathrm{~nm}$. Before and after the laser irradiation the samples were characterised by X-ray reflectometry, electron microscopy (SEM), atomic force microscopy (AFM) and laser profilometry.

\section{Results}

During oxidation two oxide layers are produced, at the surface $\mathrm{Fe}_{2} \mathrm{O}_{3}$ (thickness $\approx 20 \mathrm{~nm}$ ) and in depth $\mathrm{Fe}_{3} \mathrm{O}_{4}$ [11] (thickness $\approx 1000 \mathrm{~nm}$ ) as $\mathrm{X}$-ray reflectometry, AFM and SEM measurements show. The oxide layer exhibits a quite rough surface: its thickness is inhomogeneous $( \pm 100 \mathrm{~nm}$ around its mean value), the lateral size of the grains is around $1 \mu \mathrm{m}$.

Figure 1 shows two surfaces after laser annealing, the first one in air, the second after 40 minutes of electrochemical polarisation. In air, after 10 laser pulses at $570 \mathrm{~mJ} / \mathrm{cm}^{2}$, the laser profilometry image of the impact crater (Fig. 1a) shows that the oxide layer was not removed and the oxide surface was melted, as show by SEM (Fig. 1b). Similar results have been found when the laser interaction was performed in borate solution without an electrochemical control. In contrast to this result Fig. 1c shows an irradiated surface after 40 minutes of cathodic polarisation. After 10 laser pulses at $570 \mathrm{~mJ} / \mathrm{cm}^{2}$, 


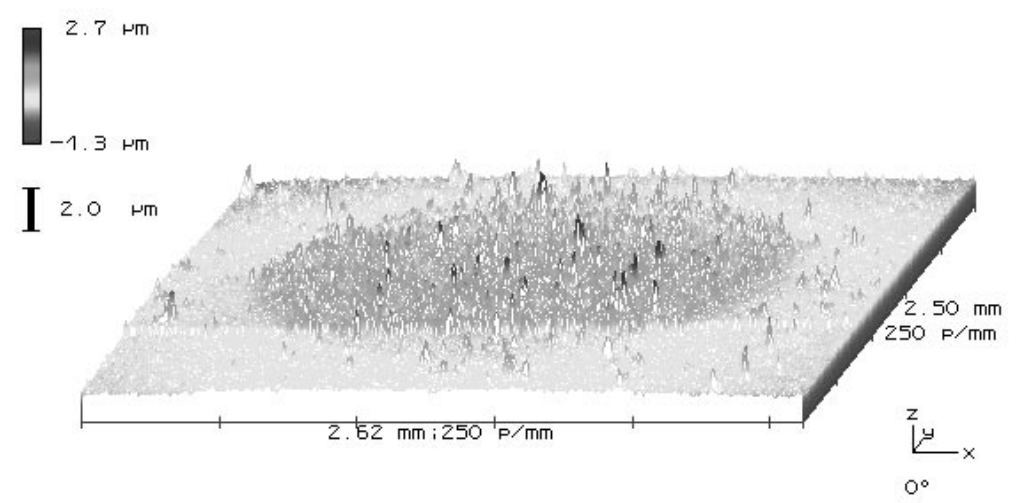

a)

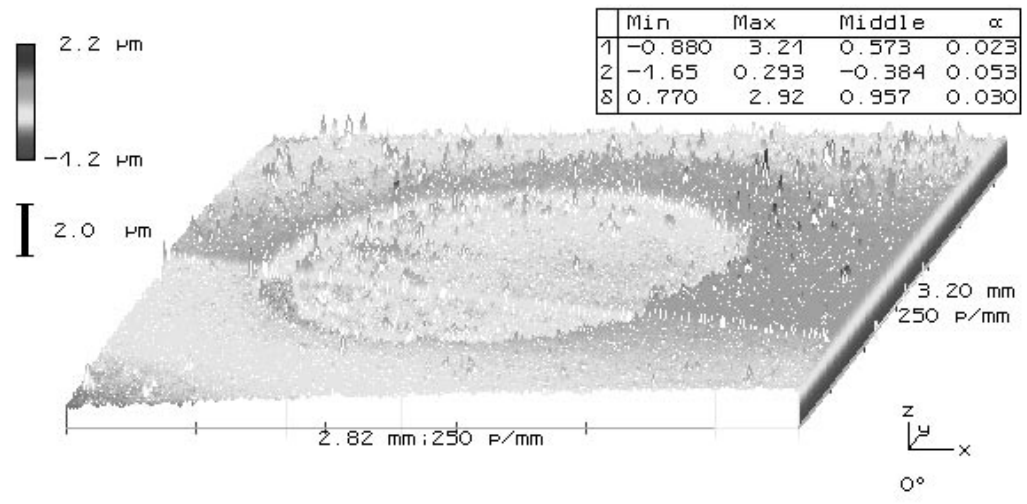

c)

b)

d)
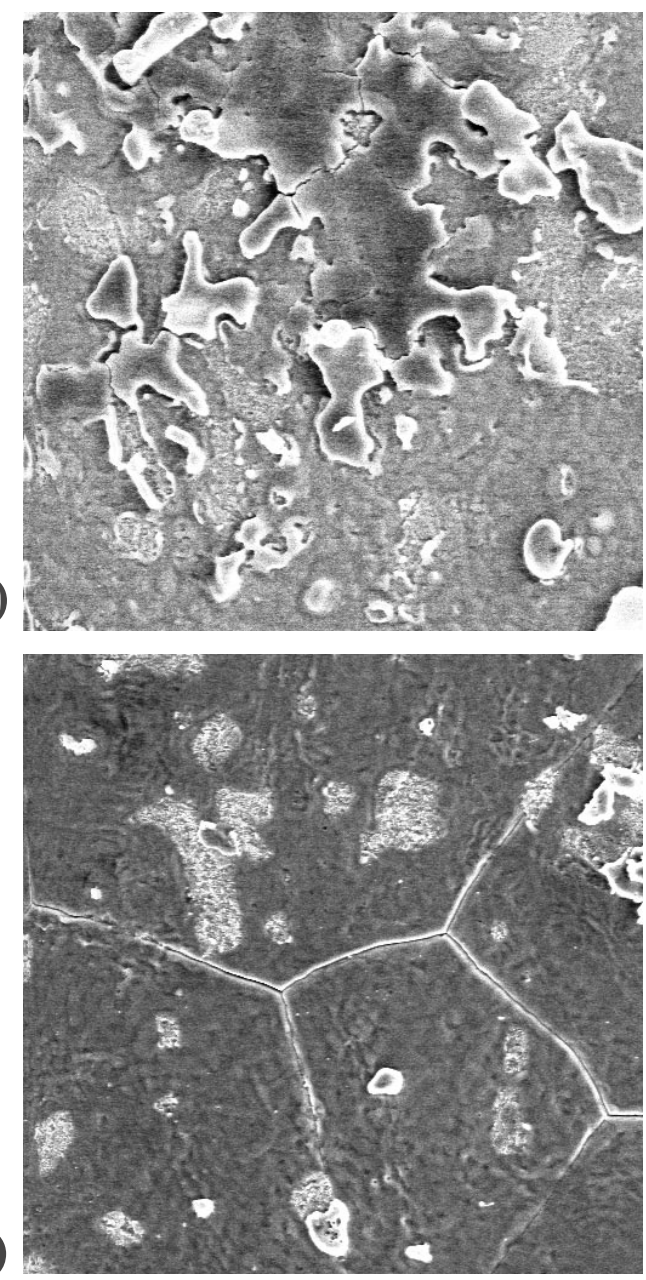

Fig. 1. a Laser profilometry image of the impact crater after irradiation in air $\left(570 \mathrm{~mJ} / \mathrm{cm}^{2}\right)$. The oxide layer is not removed. b SEM image of the impact crater after laser irradiation in air $\left(570 \mathrm{~mJ} / \mathrm{cm}^{2}\right)$. The oxide surface was melted. c Laser profilometry image of the impact crater after irradiation in borate solution with an electrochemical control $\left(570 \mathrm{~mJ} / \mathrm{cm}^{2}\right)$. The oxide layer is removed. d SEM image of the impact crater after irradiation in borate solution with an electrochemical control $\left(570 \mathrm{~mJ} / \mathrm{cm}^{2}\right)$. The oxide layer is removed

the laser profilometry image (Fig. 1c) exhibits that the complete oxide layer was removed. The surface in the crater was not melted (Fig. 1d). Consequently after 40 minutes polarisation, the oxide layer is removed efficiently without any trace of melting.

In order to explain this influence of electrochemical control on the efficiency of oxide layer removal by laser pulses, the optical properties of the oxide layer have been investigated by spectrometry at normal incidence in various environments.

Figure 2 a represents reflection spectrums of the Fe oxide surface in an electrochemical cell registered before and after 40 minutes of cathodic polarisation. In the inset of Fig. $2 \mathrm{a}$ it can be seen that the overall spectral behaviour is only slightly changed. Nevertheless a closer look in the visible range shows that beside a shift to smaller reflectivities an additional periodic behaviour appears. In order to enhance the visibility of this effect the spectrum after cathodic polarisation was normalised on the spectrum before this treatment. Therefore the spectrum in Fig. $2 b$ represents the ratio of the intensity with electrochemical control divided by the intensity without it. Now the periodic behaviour can clearly be seen. Since the periodicity is changing with wavelength it is natu- ral to take an interference effect into consideration. For that purpose a thin film model will be given in the next chapter. The result is already shown in Fig. 2b. Despite the simplicity of the model it shows a fair agreement with the experimental data.

\section{Discussion}

The optical properties of the oxide layer changed upon application of potential. The following model quantifies this behaviour. In this model the thin layer of $\mathrm{Fe}_{2} \mathrm{O}_{3}$ is neglected, as its contribution to the optical behaviour is small compared to the $1000 \mathrm{~nm} \mathrm{Fe}_{3} \mathrm{O}_{4}$ layer. Furthermore it is assumed that the layers have homogeneous optical properties throughout their thickness.

Therefore the system is composed of three layers (Fig. 3a), a substrate of pure iron with a complex refractive index $\tilde{n}_{3}=n_{3}-i k_{3}$ and sufficiently thick to be considered opaque, a oxide layer of $\mathrm{Fe}_{3} \mathrm{O}_{4}$ with a complex refractive index $\tilde{n}_{2}=n_{2}-i k_{2}$ and a thickness $(h)$ and a solution of borate with a complex refractive index $\tilde{n}_{1}=n_{1}-k_{1}$, approximate at $\tilde{n}_{1}=1.33$ (Fig. 3a) [12]. Consider an incident electromag- 

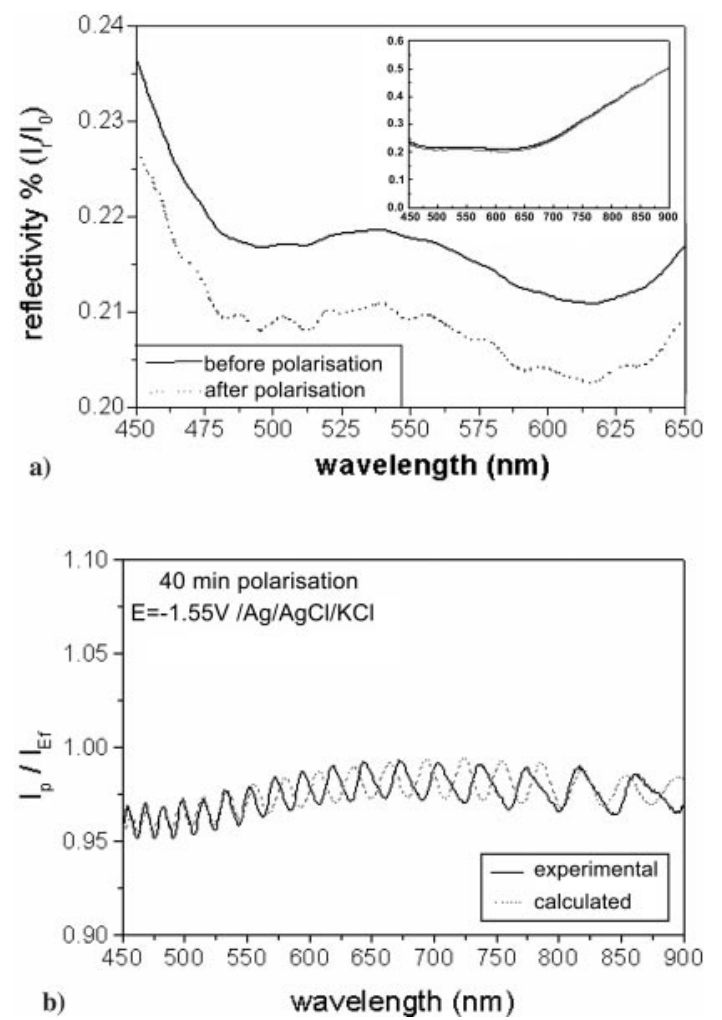

Fig. 2. a Reflected intensity before and after polarisation of the oxide layer. The inset shows the complete spectrum. The range from $450-650 \mathrm{~nm}$ is magnified. b Comparison between the normalised experimental and calculated curves

netic wave $E_{i}=E_{0} \mathrm{e}^{\mathrm{i} \omega t}$, then at normal incidence the reflected electromagnetic wave is

$E_{r}=E_{0}\left(R_{1}+\left(1-R_{1}\right) R_{2}\left(1-R_{3}\right) \mathrm{e}^{\mathrm{i} \delta} q^{-\frac{a}{2} 2 h}\right) \mathrm{e}^{\mathrm{i} \omega t}$

With $\delta=\left(4 \pi h n_{2}\right) / \lambda_{0}, \alpha=\left(4 \pi k_{2}\right) / \lambda_{0}$ and $R_{1}$ the reflectivity at the interface $1 / 2$. Further $R_{1}=\left|\left(\hat{n}_{1}-1\right) /\left(\hat{n}_{1}+1\right)\right|$ with $\hat{n}_{1}=\tilde{n}_{2} / \tilde{n}_{1}, R_{2}$ is the reflectivity at the interface $2 / 3$ with $R_{2}=\left|\left(\hat{n}_{2}-1\right) /\left(\hat{n}_{2}+1\right)\right|$ with $\hat{n}_{2}=\tilde{n}_{3} / \tilde{n}_{2}$ and $R_{3}$ is the reflectivity at the interface $2 / 1$ with $R_{3}=\left|\left(\hat{n}_{3}-1\right) /\left(\hat{n}_{3}+1\right)\right|$ who $\hat{n}_{3}=\tilde{n}_{1} / \tilde{n}_{2}$. At normal incidence the reflected intensity is

$I=\frac{c n}{4 \pi}\left|E^{2}\right|$

So

$I_{r}=I_{0}\left|R_{1}+\left(1-R_{1}\right) R_{2}\left(1-R_{3}\right) \mathrm{e}^{\mathrm{i} \delta} \mathrm{e}^{-\alpha h}\right|^{2}$

Let $a=R_{1}$ and $b=\left(1-R_{1}\right) R_{2}\left(1-R_{3}\right) \mathrm{e}^{-\alpha h}$, so the (3) become

$I_{r}=I_{0}\left(a^{2}+2 a b \cos \delta+b^{2}\right)$.

As the changes are small once again the intensities were normalised:

$\frac{I_{r \text { with polarisation }}}{I_{r \text { without polarisation }}}=\frac{a^{2}+2 a b \cos \delta+b^{2}}{a^{\prime 2}+2 a^{\prime} b^{\prime} \cos \delta^{\prime}+b^{\prime 2}}=\frac{I_{p}}{I_{E f}}$, a)
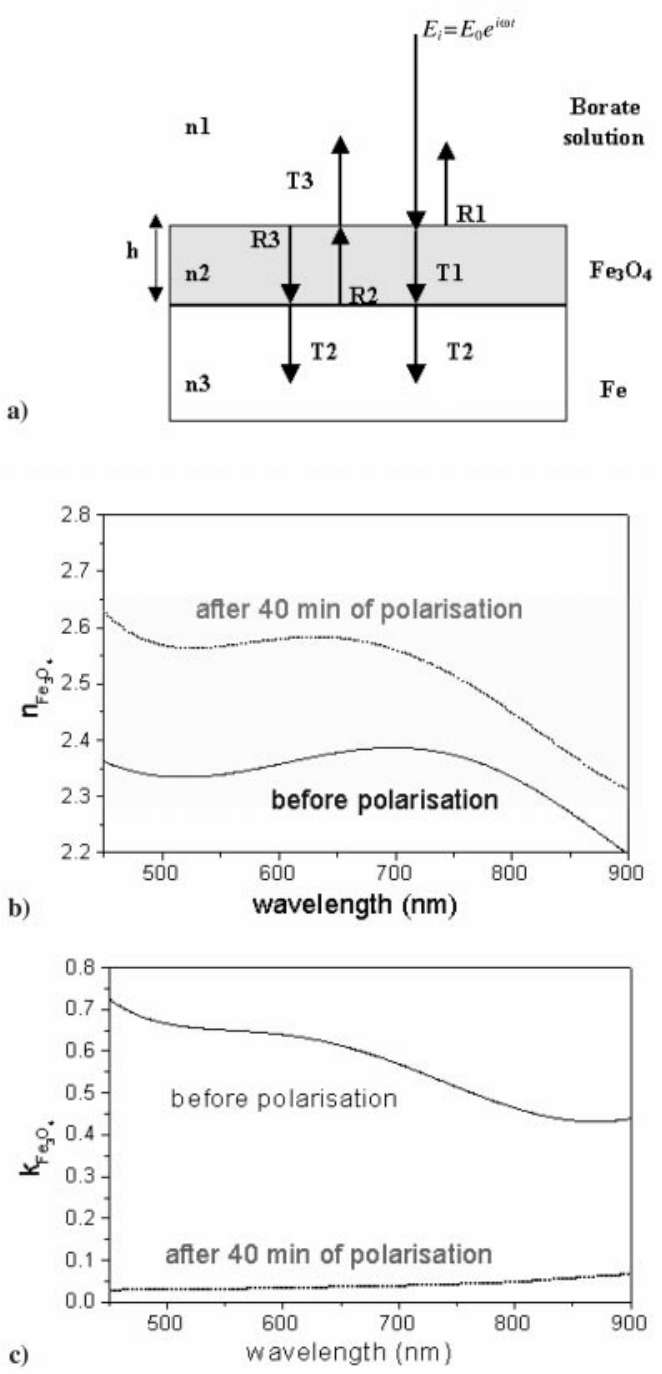

Fig. 3. a Schematic diagram of the optical model. b and c Calculated values of $n$ and $k$ of the $\mathrm{Fe}_{3} \mathrm{O}_{4}$ layer before and after polarisation

where $a, b$ and $\delta$ characterise the refractive index of $\mathrm{Fe}$ and $\mathrm{Fe}_{3} \mathrm{O}_{4}$ after polarisation and $a^{\prime}, b^{\prime}$ and $\delta^{\prime}$ the refractive index of $\mathrm{Fe}$ and $\mathrm{Fe}_{3} \mathrm{O}_{4}$ before polarisation. For the optical properties before polarisation data of [13] and [14] were used.

These theoretical considerations allow to determine the optical properties $(n, k)$ of the oxide layer after 40 minutes of an electrochemical control as function of the wavelength (Fig. 3b,c). The real part is not largely modified $(10 \%)$, but the imaginary part is reduced by a factor 10 leading to an important increase of the optical absorption length. The reason for this huge change of $\mathrm{k}$ is not clear at this moment. A possible explanation assumes that diffusion of atomic hydrogen into the oxide takes place, which is known to happen at cathodic polarisation [15]. Despite these missing details, the reason for the differences in the cleaning process are clear now: Before polarisation the laser pulses energy is deposited at the surface layer (Fig. 4a), and the oxide is melted. After polarisation, the energy is deposited in the volume of the oxide layer (Fig. 4b) which leads to a complete removal of the oxide. 


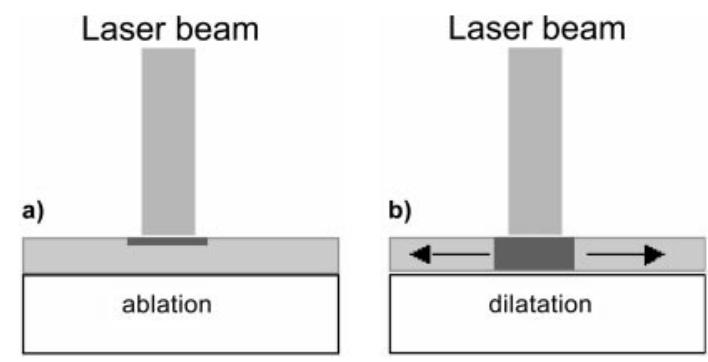

Fig. 4a,b. Schematic picture of the energy absorption in the $\mathrm{Fe}_{3} \mathrm{O}_{4}$ layer $\mathbf{a}$ before and $\mathbf{b}$ after electrochemical polarisation

\section{Conclusion}

This study has shown that laser cleaning is clearly more efficient with electrochemical control. This behaviour is explained by the modified imaginary part of the oxide layer after polarisation.

Acknowledgements. Pierre Pasquet and Raul del Coso are doctoral students in the framework of the TMR ERB FMRX CT098-0188 "Laser Cleaning"

\section{References}

1. P.E. Pehrsson, R. Kapla: J. Mater. Res. 4, 1480 (1989)

2. J. Xieet, A. Ka: J. Appl. Phys. 82, 4745 (1997)

3. A.A. Shul'ginov: Sov. Phys. Acoust. 37, 421 (1991)

4. Y.F. Lu, M.H. Hong, S.J. Chua: J. Appl. Phys, 79, 2186 (1996)

5. Y.F. Lu, M. Takai, T. Shiokawa, Y. Aoyagi: Appl. Phys. A 33, 324 (1994)

6. O. Yavas, P. Leiderer, H.K. Park, C.P. Grigoropoulos, C.C. Poon, A.C. Tam: Phys. Rev. Lett. 72, 2021 (1994)

7. R. Oltra, O. Yavas, F Cruz, J P Bouquillon, C Sartori: Appl. Surf. Sci. 96-98, 484 (1996)

8. R. Oltra, O. Yavas, O. Kerrec: Surf. Coat. Technol. 88, 151 (1996)

9. R. Oltra, O. Yavas, O. Kerrec: Appl. Phys. A, Mater. Sci. Proc. 64, 321 (1996)

10. I.G. Uzlov, V.L. Spivakov, E.A. Shpak: Metal Sci. Heat Treatment 39, 19 (1997)

11. J.C. Colson, R. Oltra, P. Pasquet: High Temperature Corrosion and Materials Chemistry, Vol. 98-9, ed. by P.Y. Hou, M.J. McNallan, R. Oltra, E.J. Opila, D.A. Shores, The Electrochemical Society (1998) p. 118

12. M. Born, E. Wolf: Principles of optics: electromagnetic theory of propagation interference and diffraction of light, 6th edn. (1993)

13. W.F.J. Fontijn, P.J. van des Zaag, M.A.C. Devillers, V.A.M. Brabers, R. Metselaar: Phys. Rev. B 56, 5432 (1997)

14. Handbook of Laser Science and Technology, Vol IV (CRC Press, Boca Raton, Floride Optical Materials 1996)

15. T.-Y. Zhang, Y.-P. Zheng, Q.-Y. Wu: J. Elec. Soc. 146, 1741 (1999) 